\title{
III. On the general expression for the sum of an infinite geometrical series
}

\section{J.R. Young}

To cite this article: J.R. Young (1846) III. On the general expression for the sum of an infinite geometrical series, Philosophical Magazine Series 3, 28:184, 10-12, DOI: $10.1080 / 14786444608645344$

To link to this article: http://dx.doi.org/10.1080/14786444608645344

曲 Published online: 30 Apr 2009.

Submit your article to this journal

Џ Article views: 4

Q View related articles $\square$ 
it is no wonder that they should be so, when we consider how vastly greater must be the force required to uplift the rocky crust of the earth and wrench it asunder, than that which will support a column of water equal to the thickness of that crust.

Since the foregoing paper was read, I have rather hastily examined some othei portions of water taken from different pseudomorphous crystals. One of those portions contained muriatic and sulphuric acids, iron, a trace of lime, and of common salt. Acid was a little in excess, and some peroxide of iron was left in the cavity from which the water was taken. In another the same acids were detected and some iron. In the third portion there seemed to be nothing besides a little common salt. In many of the octahedral cavities, oxide of iron was found, and sometimes iron pyrites or copper pyrites adhering to the sides; these were apparently deposited from some of the water which had entered the crystals in some instances, but in others they were evidently imbedded in the fluor, and, adhering to the deposit of quartz, were not dissolved with the former.

Earthy carbonate of iron occurs in some cavities mixed with very minute crystals of quartz; and I have one pseudomorphous quartz crystal which is filled with fragments of fluor, intermixed with translucent fragments of carbonate of iron and earthy carbonate of iron, all curiously cemented together into one mass; the iron ore being rather in excess.

I have also some hollow pseudomorphous crystals of quartz formed originally on carbonate of iron, which appear to be water-tigtst, and yet the latter substance has, like the fluor, been abstracted.

III. On the General Expression for the Sum of an Infinite Geometrical Series. By J. R. Young, Professor of Mathematics in Belfast College*.

T THE general expression for the sum of the infinite series

$$
1-x+x^{2}-x^{3}+x^{4}-\& \mathrm{c} \text {. }
$$

is

$$
\mathrm{S}=\frac{1}{1+x}-\frac{x^{\infty \prime \prime}}{1+x}
$$

which reduces to $\frac{1}{1+x}$ when $x$ is a proper fraction, either po-

* Communicated by the Author. 
sitive or negative, on account of the evanescence of $x^{\infty \prime \prime}$. It is usual to consider the infinite exponent in this expression as invariable throughout all the changes of $x$ within the limits 0 and 1 ; although it is known that for any fixed exponent short of infinite, however great it may be, the expression into which it enters becomes more and more considerable as $x$ advances from 0 towards 1 ; and notwithstanding the additional fact, that when this exponent is actually infinite, the expression referred to becomes ultimately equal to $\frac{1}{e}$.

But it is evident-due weight being given to the circumstances here mentioned-that this assumption, as to the invariability of the infinite exponent, is unwarrantable and erroneous; and that the exponent must follow some law of variation exactly fitted to counteract and neutralize the tendency which, as $x$ approaches to 1 , the expression $x^{\infty}$ " would otherwise have to depart from zero, and ultimately to become $\frac{1}{e}$. If $x$, at any stage of its approach to 1 , be generally represented by $1-\frac{1}{k}$, then the law of variation alluded to will be expressed by $\infty^{\prime \prime}=l c \infty^{\prime}$ : that is, the exponent must vary as k. For it is a remarkable fact that, commencing with the exponent 4 and proceeding onwards to infinity, we shall invariably have

$$
\begin{aligned}
& \left(\frac{3}{4}\right)^{4}=\cdot 3 \ldots,\left(\frac{4}{5}\right)^{5}=\cdot 3 \ldots,\left(\frac{5}{6}\right)^{6}=\cdot 3 \ldots,\left(\frac{6}{7}\right)^{7}=\cdot 3 \ldots, \\
& \left(\frac{16801}{16802}\right)^{16802}=\cdot 3 \ldots, \ldots\left(\frac{25684}{25685}\right)^{25685}=\cdot 3 \ldots, \ldots\left(1-\frac{1}{\infty}\right)^{\infty}=\cdot 3 \ldots
\end{aligned}
$$

And since $(\cdot 3 \ldots)^{\infty}$ is necessarily zero, and no power short of infinite can give zero, it follows that in order that $\left(1-\frac{1}{k}\right)^{\infty \prime \prime}$ may be uniformly zero, and that all tendency to depart from zero may be counteracted, $\infty$ " must be $k \infty^{\prime}$; so that the strictly accurate form for $\mathrm{S}$ is

$$
\mathrm{S}=\frac{1}{1+\left(1-\frac{1}{k}\right)}-\frac{\left(1-\frac{1}{k}\right)^{k \infty}}{1+\left(1-\frac{1}{k}\right)}
$$

which is equal to $\frac{1}{2}$ when $k$ is infinite. And in this manner is the formula, employed in my paper (p. 363, last vol.), established. 
In the same way that it has now been proved that $\left(1-\frac{1}{k}\right)$ is always equal to $3 \ldots$, whatever be $k$, above 3 , may it be further shown that $\left(1+\frac{1}{k}\right)^{k}$ is always equal to $2 . .$. ; and thence that $\left(1+\frac{1}{k}\right)^{k \infty \prime}$ is necessarily infinite when $k$ is: so that it is indisputably true that the extreme of the convergent cases of the above series $S$, usually written in the form

$$
1-1+1-1+1-1+\& c \text {. }
$$

is $\frac{1}{2}$, and that the extreme of the divergent cases, usually written in the same form, is really infinite, as stated in my former paper; which last conclusion could never have been anticipated from the theory hitherto prevalent. The views now developed are only the continuation and completion of those exhibited in my paper on Series submitted to the British Association in June 1845. If I have been anticipated in any of these views, which are doubtless calculated to produce a reform in the existing theory, I hope to be informed of the circumstance through the medium of this Journal. I have only further to add, that when an expression for the convergent cases of a series is found-as it often may be by aid of the differential theorem-then the general equivalent of the scries may afterwards be ascertained by developing this expression sufficiently far to unfold to us the general form of the remainder. The expression for the convergent cases of the general series, discussed at page 439 of the last volume, may in this manner be determined; and the development of this expression by common division, as there proposed, furnishes the formula by which that expression must be corrected, in order that the algebraical equivalent of the series may be exhibited in its utmost generality.

Belfast, November 21, 1845.

IV. On the Conversion of Cane-sugar into a substance isomeric with Cellulose and Inuline. By Thomas Tilley, Esq., Ph.D., and Dovglas Maclagan, M.D., F.R.S. Edin.**

WHEN the juice of beetroot undergoes fermentation at temperatures yarying from $30^{\circ}$ to $40^{\circ} \mathrm{C}$, the canesugar which it contains is at first converted into sugar of grapes, and after some time into mannite, lactic acid and a

* Communicated by the Chemical Society; having been read April 21, 1845. 